\title{
Robotics Enabled Autonomous and Closed Loop Trauma Care in a Rucksack
}

\author{
Ronald K. Poropatich, MD ${ }^{1, *}$ and Michael R. Pinsky, MD²
}

\begin{abstract}
Artificial intelligence (Al) plays a critical role across all military programs of the department of defense (DoD) and is a key strategy to maintain technical superiority to peer and near-peer adversaries. Similar to the civilian sector, the role of Al in healthcare is also being actively pursued by the DoD and is incorporated in the Medical Robotics and Autonomous Systems programs. This article describes a recently funded DoD program at the University of Pittsburgh that aims to develop a prehospital robotically controlled autonomous cardiopulmonary resuscitation platform that is small and portable and planned for battlefield delivery to casualties through either manned or unmanned air or ground vehicles. The research program is called TRAuma Care In a Rucksack (TRACIR) and its goals are to develop critical care decision support algorithms based on a large prehospital data set of medical casualty trauma evacuations in Pittsburgh overlaid with $\mathrm{Al} /$ machine learning using functional hemodynamic monitoring principles that will robotically drive critical care interventions. The TRACIR program and its research aims are described.
\end{abstract}

\section{Introduction}

Defense department establishment of artificial intelligence programs

Transformative uses of artificial intelligence (AI) today are founded in federal government investments in fundamental AI research that reach back over decades. Building the foundations of tomorrow's AI innovations will require new interdisciplinary collaborations, resources and strategic vision.

In February 2019, President Donald Trump signed an executive order expanding the administration's efforts to foster the research and development of AI tools in government. ${ }^{1}$ The executive order directs agency heads to set aside research and development funding in their fiscal 2020 budget requests and elevate $\mathrm{AI}$ as a national priority. This coordination function is crucial to the emerging AI arms race with Russia and China. ${ }^{2}$

In June 2018, the defense department launched the Joint Artificial Intelligence Center (JAIC) focusing on agility and speed to meet the needs of not only the warfighter but also back-office functions. ${ }^{3}$ The JAIC is a key component of the National Defense Strategy that aims to maintain our strategic position, prevail on the future battlefields, and safeguard free and open international borders. ${ }^{4}$ The JAIC will be the main artery delivering AI governance, tools, standards, and more to all military services and department of defense (DoD) agencies with near-term transitioning from research and development to operational fielded capabilities.

The Army's Futures Command (AFC) was established in August 2018 and represents the most significant army reorganization effort since 1973, when Forces Command and Training \& Doctrine Command were established. ${ }^{5}$ The AFC leads the army's future force modernization enterprise by assessing the future operational environment, emerging threats, and new technologies to develop and deliver concepts, requirements, future force designs, and modern materiel solutions to meet soldiers' wartime needs.

In February 2019, the AFC established the AI task force whose initial priorities are for applications of AI to equipment maintenance/logistics; situational awareness; medical and humanitarian assistance; and disaster relief. ${ }^{6}$ The AI task force is located at Carnegie Mellon University (CMU) in Pittsburgh and augments the U.S. army's long-standing commitment to modernization and future technology, while also strengthening its ties to fundamental

${ }^{1}$ Center for Military Medicine Research and ${ }^{2}$ Department of Critical Care Medicine, University of Pittsburgh, Pittsburgh, Pennsylvania. 
research in academia. The AFC AI task force works in close coordination with the JAIC for current collaborations for DoD-funded projects of $\mathrm{AI}$ in healthcare.

\section{Military medical use of Al on the battlefield}

The growing use of unmanned systems and robotics on the future battlefield affords both great opportunities and challenges to far-forward medical operations, especially in the U.S. army's concept for multi-domain battle. Multi-domain battle is an operational concept with strategic and tactical implications that deliberately focuses on increasingly capable adversaries who challenge deterrence and pose strategic risk to U.S. interests. The multi-domain battle concept describes how U.S. and partner forces organize, practice, and employ capabilities and methods across domains, environments, and functions over time and physical space to contest these adversaries in armed conflict. ${ }^{7}$

Recent military conflicts in Southwest Asia and Africa have resulted in challenges to evacuate a casualty to a medical facility for definitive care. At times, this has required service members to treat in place and render "prolonged field care" until medical evacuation can be performed. Prolonged field care (PFC) is now a military reality and includes field medical care, applied beyond "doctrinal planning time-lines" to decrease patient mortality and morbidity. The location for PFC on the battlefield does not directly correspond to traditional echelons of care and will most likely be in remote/austere (Africa, regions of Southwest Asia) or difficult-to-reach (urban warfare) locations with limited access to traditional medical logistics resources. PFC will need to utilize limited resources, with current planning requiring novel logistic solutions (i.e., drone delivery of blood/medical products or other medical capabilities) for sustainment of care until the patient arrives at the next appropriate level of care. ${ }^{8}$

Hemorrhage remains the primary cause of preventable death on the battlefield and will become more problematic during PFC settings as anticipated for future multidomain battle situations. Hemorrhage control is multifactorial and starts with point-of-injury care. During situations with $\mathrm{PFC}$, interventions in the prehospital environment and perioperative period affect outcomes. Timely and appropriate therapies impact survival. Novel solutions for hemorrhage control-such as unmanned drone delivery of whole blood or blood component therapy from a sustaining base in theater-have the potential to mitigate the deleterious outcomes for hemorrhage (both compressible and noncompressible torso hemorrhage).

To help address the military need for far-forward logistics and PFC, the Defense Advanced Research Project Agency (DARPA) in 2009 began the transformer pro- gram that sought solutions to provide more frontline military units with mission-tailored vertical takeoff and landing (VTOL) capabilities for logistics/cargo resupply delivery and potential casualty evacuation (CASEVAC) using drones. ${ }^{9}$ In 2013, DARPA selected the Aerial Reconfigurable Embedded System (ARES) design concept to operate as an unmanned platform capable of transporting a variety of payloads (Fig. 1) The ARES VTOL flight module would travel between its home base and field operations to deliver and retrieve several different types of mission modules, each designed for a specific purpose (i.e., intelligence, surveillance and reconnaissance, cargo resupply, and casualty evacuation). Twin tilting ducted fans would provide efficient hovering and landing capabilities in a compact configuration, with rapid conversion to high-speed cruise flight. ${ }^{9}$ The DoD is actively seeking technical drone solutions that meet a variety of military battlefield needs. In addition to DARPA and other DoD organizations, the Army Research Laboratory is working to incorporate AI-based scene perception capabilities into drones and thermal recognition technology to give warfighters increased situational awareness. ${ }^{10}$

\section{TRAuma Care In a Rucksack overview}

TRAuma Care In a Rucksack (TRACIR) was initially conceptualized in 2016 and represents a disruptive technology proposed to create a paradigm shift in how medical data are defined, stored, captured, visualized, and shared such that a more easily transportable semiautonomous and autonomous tactical combat casualty care (TCCC) solution can be developed. ${ }^{11}$ The University of Pittsburgh and CMU in Pittsburgh have partnered toward development of an autonomous robotic enabled cardiopulmonary resuscitation platform with a form factor that will fit in a rucksack and placed on unmanned air or ground vehicles to support casualty emergent PFC and evacuation missions. The multidisciplinary multiorganization research team brings together the practical clinical expertise of novel diagnostic and treatment approaches of the University of Pittsburgh School of Medicine (Trauma, Critical Care, Emergency Medicine) with the machine learning and advanced robotics expertise of CMU and autonomous systems and decision-making expertise at University of Arizona. The TRACIR project was funded by the AFC in Spring of 2019 and aims to advance PFC and battlefield medicine by using large prehospital data sets overlaid with AI tools to develop clinical decision support tools in prehospital settings.

\section{TRACIR methods}

The research project includes an approved institutional review board protocol for both animal and human use 


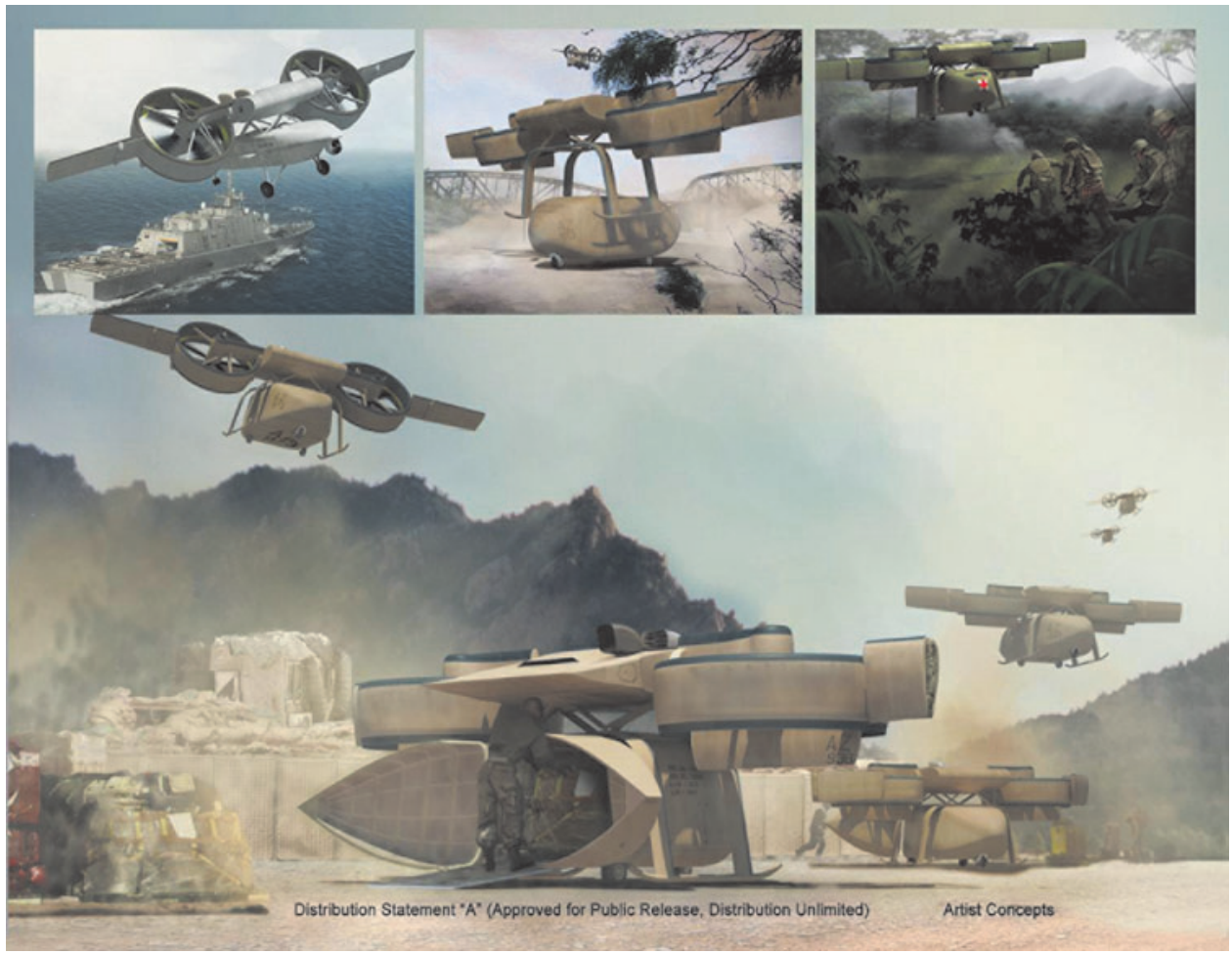

FIG. 1. DARPA Aerial Reconfigurable Embedded System (ARES) - Novel Unmanned Drone Capabilities for Future Healthcare Delivery.

for a cardiopulmonary resuscitation approach based on dynamic measures of arterial pressure and its surrogate measures during breathing and hydrostatic maneuvers. This approach uses functional hemodynamic monitoring (FHM) as developed by Michael R. Pinsky, MD, the scientific principal investigator of the TRACIR program linked to a closed loop controller. However, to personalize these proven protocols to the young trauma population, we need to link these response profiles to real response profiles of trauma patients. We have such a large database that we will data mine for this purpose.

The University of Pittsburgh Medical Center (UPMC) has 17 medevac helicopters over a four-state region that have transported $>240,000$ medevac patients to UPMC for the past 20 years-averaging 13,000 transfers per year. An extensive prehospital database (StatMedEvac) including high-definition waveform physiologic data is already in use with numerous other funded DoD prehospital research studies.

The TRACIR project is funded to utilize AI/machine learning to data mine the existing high-granularity (waveform, $250 \mathrm{~Hz}$ ) physiologic monitoring data as part of the StatMedEvac clinical database of $>7000$ emergency air transports of young trauma patients (age 18-45 years) who were transported directly from a trauma site to the UPMC Level 1 Trauma Center. This project will integrate the 7000-prehospital trauma patient data set and combine it with the corresponding in-patient hospital records to create an anonymized comprehensive big data repository that stitches together prehospital data with the corresponding in-patient data for each of the trauma subjects. This large StatMedEvac/in-patient trauma database with applied machine learning methodology will give rise to an intelligent interpretation of multiple biosignals collected from our clinically relevant trauma models (traumatized swine studies followed by human studies) to define shock severity and autonomously drive these FHM protocols through hard and soft robotics interventions. The planned device will perform continuous monitoring to recognize software rule-based polytrauma events and drive specific FMH protocols autonomously followed by robotically controlled critical care interventions. The TRACIR-derived predictive algorithm will be developed in our swine hemorrhagic shock model followed by human studies, with the physiologic monitoring provided by novel FDA-approved biosensors used currently in hospital critical care settings (electrical inductance tomography, small arteriole vasoconstriction, plethysmographic variability index, etc.). Human subjects will then be instrumented with these new noninvasive biosignal devices and placed in a lower body negative pressure chamber applied to simulate hemorrhage at varying degrees of severity and enable further 
refinement of the TRACIR predictive algorithm. This iterative development of the algorithm will be further tested and refined in our UPMC Emergency Medicine Department in traumatized patients and compared with usual care.

As the University of Pittsburgh team develops the autonomous predictive cardiopulmonary resuscitation software, it is working in parallel with a separately funded DoD complementary project comprising a multidisciplinary team of scientists and robotics experts at CMU to build the hard and soft robotic solutions to enable autonomous prehospital critical care interventions (i.e., vascular access, image detection of pneumothorax and noncompressible torso hemorrhage, and airway management). The TRACIR program will also include iterative development of a sensorized bioelectric wrap with embedded imaging sensors to interrogate the casualty to better assess for trauma. Additional TRACIR development will include visual display capability for providers and ultimately miniaturization of component parts to conform to a size (cube and weight) and power demand that could fit in a rucksack and be deployed to a remote site. A TRACIR systems overview is depicted in Figure 2.

\section{TRACIR research aims}

Aim 1. To develop an autonomous, closed loop, and integrated multimodal sensor system to inform monitoring and resuscitation for trauma care in the field using machine learning algorithms to automate fluid resuscitation, ventilation, and stabilization that enables early intervention for prolonged trauma care based on FHM principles.

Aim 2. To develop hard and soft robotic solutions to facilitate mechanical stabilization of patients, control application of sensors, and enable automated physical (pressure controlled, palpable, and tactile) diagnostics and therapeutics in the field for unmanned air and ground/ CASEVAC mission support capabilities.

Aim 3. To develop embedded ultrasound sensors in a soft robotics cervical collar and torso wrap to assess for pneumothorax and hepatosplenial laceration that result in appropriate critical care interventions (needle thoracostomy and venous access).

\section{Results}

At the time of this writing, the DoD awards to both the University of Pittsburgh and CMU were completed. Preliminary studies were initiated in June 2019.

\section{Discussion}

The TRACIR project addresses many of the combat casualty care operational deficiencies identified in past conflicts by proposing a novel approach to a fundamental technical challenge. This challenge is to overcome the

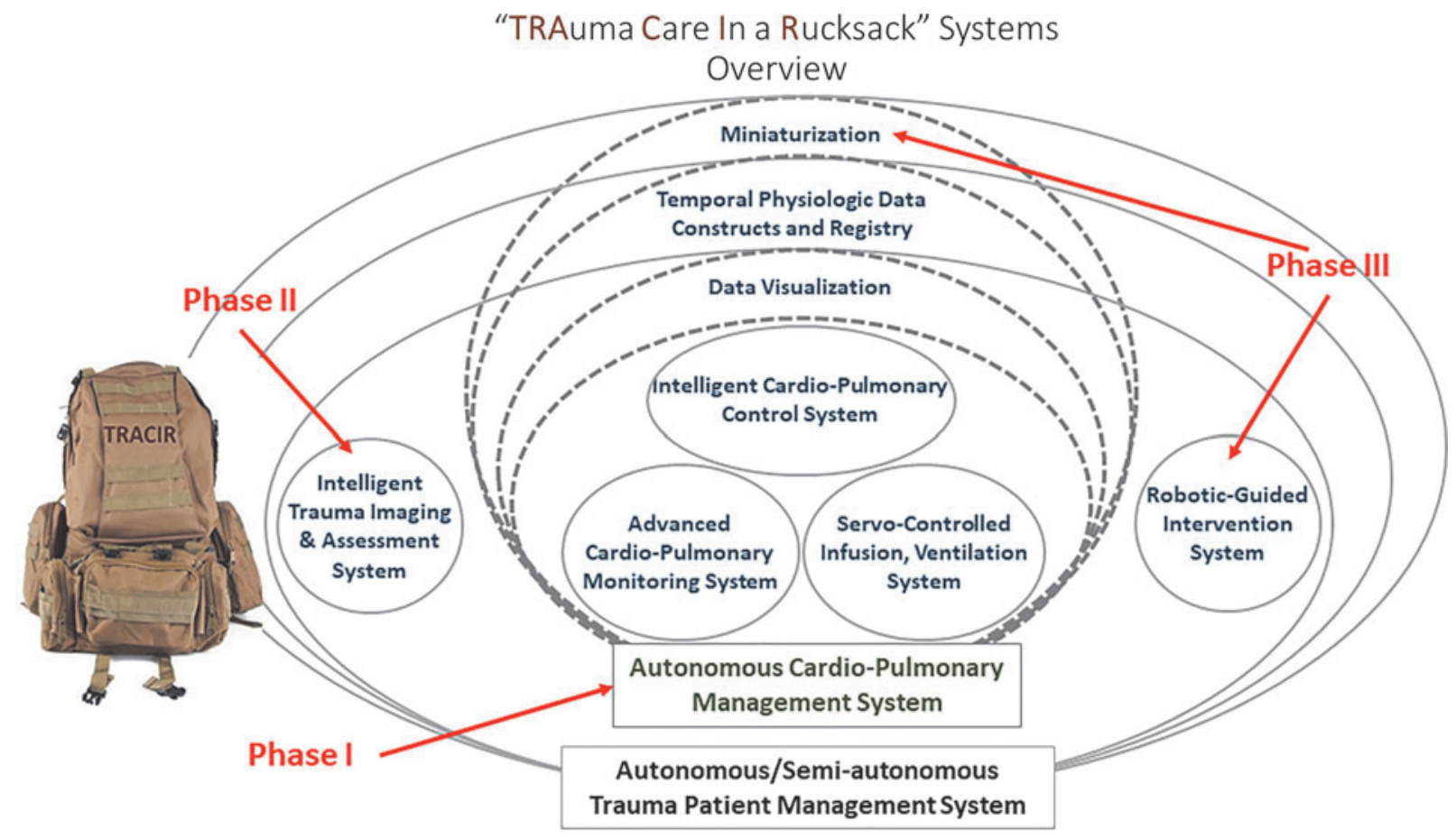

FIG. 2. TRACIR Provides Next Generation Intelligent Cardiopulmonary Resuscitation Management Tool at its Core with Advanced Robotics Used to Aid in Providing Autonomous Assessment and Interventions. 
pervasive use of subjective physiologic signs and symptoms used as the standard of care to triage or assess and treat patients that is also an obstacle toward providing a semiautonomous and autonomous TCCC solution. The proposed TRACIR platform satisfies key operational requirements for use by any vehicle of opportunity, during PFC in denied environments, and for future unmanned aerial systems' needs. Creating smart alarms linked to intelligent clinical decision support software is revolutionizing acute in-hospital care that will reduce the cognitive stress of patient care. Moving this AI format to out-ofhospital environments represents the logical evolution of these approaches into these military-relevant domains.

TRACIR is a 4-year DoD-funded research project led by the University of Pittsburgh and highlights the extensive partnerships with robotic entities, to include the National Robotics Engineering Center at CMU and the DoD-funded Advanced Robotics Manufacturing Institute, both located in Pittsburgh, PA, coupled with the autonomous decision-making expertise at the University of Arizona. By fusing data captured from multiple sensors and applying supervised machine learning, we are developing more predictive cardiopulmonary resuscitation events than those currently exist and are applying them to hard and soft robotic solutions in a form factor to fit in a rucksack. In addition to the military need, the civilian applications of TRACIR (i.e., skilled nursing facilities and wilderness medicine) are numerous and will help accelerate the commercial development.

\section{Acknowledgments}

The authors thank collaborators from the University of Pittsburgh (Frank Guyette, Leonard Weiss, David Salcido, and Giles Clermont), Carnegie Mellon University (Artur Dubrawski, Howie Choset, and Chris Atkeson), and the University of Arizona (Wolfgang Fink).

\section{Authors' Contributions}

Both Drs. Poropatich and Pinsky actively contributed to this article. Dr. Poropatich provided the necessary military background, medical need, and relevance in battlefield set- tings. Dr. Pinsky provided the scientific development of the program to include animal and human use studies.

\section{Author Disclosure Statement}

No competing financial interests exist.

\section{Funding Information}

The authors thank the U.S. Army Medical Research and Development Command for funding this project—award no. W81XWH19C0101.

\section{References}

1. Heckman J. Trump signs executive order fostering artificial intelligence R\&D in government. Federal News Network 2019. https://federal newsnetwork.com/artificial-intelligence/2019/02/trump-signs-executiveorder-fostering-ai-rd-in-government/. (Last accessed on July 31, 2019).

2. Freedberg SJ. US needs new strategy to combat Russian, Chinese 'political warfare'. Breaking Defense 2018. https://breakingdefense.com/ 2018/05/us-needs-new-strategy-vs-russian-chinese-political-warfarecsba/. (Last accessed on July 31, 2019).

3. Shanahan P. Deputy Secretary of Defense Memorandum: Establishment of the Joint Artificial Intelligence Center. 2018. https://admin.govexec .com/media/establishment_of_the_joint_artificial_intelligence_center _osd008412-18_r....pdf. (Last accessed on July 31, 2019).

4. Miler J. DoD rips wrapping paper off of new Joint Al Center. Federal News Network 2019. https://federalnewsnetwork.com/artificial-intelligence/ 2019/02/dod-rips-wrapping-paper-off-of-new-joint-ai-center/. (Last accessed on July 31, 2019).

5. Roper DS, Grassetti J. Seizing the high ground-United States Army Futures Command, Institute of Land Warfare at the Association of the United States Army 2018. https://www.ausa.org/sites/default/files/ publications/SL-18-4-Seizing-the-High-Ground-United-States-ArmyFutures-Command.pdf (Last accessed on August 5, 2019).

6. Carnegie Mellon Hosts Activation of U.S. Army AI Task Force. CMU News 2019. https://www.cmu.edu/news/stories/archives/2019/february/ army-ai-task-force.html. (Last accessed on July 31, 2019).

7. Multi-Domain Battle: Evolution of Combined Arms for the 21st Century 2025-2040. 2017. https://www.tradoc.army.mil/Portals/14/Documents/ MDB_Evolutionfor21st\%20(1).pdf. (Last accessed on August 5, 2019).

8. Keenan S, Reisberg J. Prolonged field care: beyond the "golden hour." Wilderness Environ Med. 2017; 28:S135-S139.

9. DARPA. ARES Aims to provide more front-line units with mission-tailored VTOL capabilities. 2014. https://www.darpa.mil/news-events/2014-0211 (Last accessed on July 29, 2019).

10. Williams LC. Army Research Laboratory explores Al-enhanced drones for the battlefield. 2019. https://defensesystems.com/articles/2019/04/03/ arl-drone-ai.aspx (Last accessed on August 5, 2019).

11. Berkow J, Poropatich R. TRAuma Care in a Rucksack (TRACIR), a disruptive technology concept. Small Wars J. 2016. https://smallwarsjournal.com/ jrnl/art/\%E2\%80\%9Ctrauma-care-in-a-rucksack\%E2\%80\%9D-tracir-adisruptive-technology-concept (Last accessed on February 4, 2020). 